Time Distortions in Mind 
A. Vatakis and M.J. Allman - 9789004230699 Downloaded from Brill.com04/26/2023 12:39:33PM via free access 


\section{Time Distortions in Mind}

Temporal Processing in Clinical Populations

Edited by

A. Vatakis

M.J. Allman

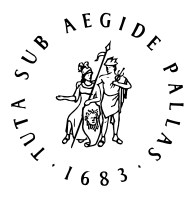

B R I L L

LEIDEN | BOSTON 
This is an open access title distributed under the terms of the Creative Commons Attribution-Noncommercial 3.0 Unported (CC-BY-NC 3.0) License, which permits any non-commercial use, distribution, and reproduction in any medium, provided the original author(s) and source are credited.

\section{Cover design: Özlem Altin}

\section{Library of Congress Cataloging-in-Publication Data}

Time distortions in mind : temporal processing in clinical populations / edited by A. Vatakis (Cognitive Systems Research Institute, Athens, Greece), M. Allman (Michigan State University, East Lansing, USA). pages $\mathrm{cm}$ Includes bibliographical references and index.

ISBN 978-90-04-23064-4 (hardback : alk. paper) -- ISBN 978-90-04-23069-9 (e-book) 1. Time perception. 2. Cognitive psychology. I. Vatakis, Argiro, editor. II. Allman, Melissa J., editor.

\section{$\mathrm{BF}_{4} 68 . \mathrm{T}_{547} 2015$ \\ $153 \cdot 7^{\prime} 53^{--}$dc23}

2015010275

This publication has been typeset in the multilingual "Brill" typeface. With over 5,100 characters covering Latin, IPA, Greek, and Cyrillic, this typeface is especially suitable for use in the humanities.

For more information, please see www.brill.com/brill-typeface.

ISBN 978-90-04-23064-4 (hardback)

ISBN 978-90-04-23069-9 (e-book)

Copyright 2015 by the Editor[s] and Authors.

This work is published by Koninklijke Brill NV. Koninklijke Brill NV incorporates the imprints Brill, Brill Hes \& De Graaf, Brill Nijhoff, Brill Rodopi and Hotei Publishing.

Koninklijke Brill NV reserves the right to protect the publication against unauthorized use and to authorize dissemination by means of offprints, legitimate photocopies, microform editions, reprints, translations, and secondary information sources, such as abstracting and indexing services including databases. Requests for commercial re-use, use of parts of the publication, and/or translations must be addressed to Koninklijke Brill NV.

This book is printed on acid-free paper. 


\section{Contents}

Preface VII

List of Figures XII

List of Tables XXII

1 Distorted Multisensory Experiences of Order and Simultaneity 1 Argiro Vatakis and Alexandra Elissavet Bakou

2 Abnormal Timing and Time Perception in Autism Spectrum Disorder?

A Review of the Evidence 37

Melissa J. Allman and Christine M. Falter

3 A Social Timing Model of Autism, Informed by Typical Development 57 Dawn Wimpory

4 Time Processing in Schizophrenia 93

Deana B. Davalos and Jamie Opper

5 Sense of Time Continuity: Possible Mechanisms of Disruption in Schizophrenia 115

Anne Giersch, Laurence Lalanne, Mitsouko van Assche, Patrick E.

Poncelet, and Mark A. Elliott

6 Predictive Timing for Rhythmic Motor Actions in Schizophrenia ${ }_{13} 6$

Yvonne Delevoye-Turrell, Hélène Wilquin, and Mariama Dione

7 Interactions of Timing and Motivational Impairments in

Schizophrenia 168

Ryan D. Ward, Billur Avlar, and Peter D. Balsam

8 Timing in Neurogenerative Disorders of the Basal Ganglia 190 Deborah L. Harrington and Stephen M. Rao

9 Timing in the Cerebellum and Cerebellar Disorders $\quad 226$ Rebecca M. C. Spencer 
10 Striatal and Frontal Pathology: Parkinson's Disease and Patients with

Lesions of the Basal Ganglia and Frontal Cortex $25^{0}$

Catherine R.G. Jones and Marjan Jahanshahi

11 Bayesian Models of Interval Timing and Distortions in Temporal Memory as a Function of Parkinson's Disease and Dopamine-Related Error Processing 281

Bon-Mi Gu, Anita J.Jurkowski, Jessica I. Lake, Chara Malapani, and Warren H. Meck

12 Aphasia as a Temporal Information Processing Disorder 328 Elzbieta Szelag, Aneta Szymaszek and Anna Oron

13 How Could Circadian Clock Genes influence Short Duration Timing? 356 Brad Nicholas

Index $\quad 383$ 


\section{Preface}

Examinations of aspects of temporal processing in clinical populations may inform not only typical psychological functioning - but may also elucidate the psychological consequences of any pathophysiological differences in temporal processing. This line of scientific enquiry may improve understanding, and potential remediation, of the psychological condition.

Humans are equipped with the remarkable ability to estimate event duration, recall past events, remember to execute future tasks, and perceive a multisensory and temporally unified world. This ability enables us (and other animals) to anticipate, learn, and adapt to temporal regularities and dynamics in the social and non-social environment. Moreover, the perception of time, typically (although not always) reveals hallmarks shared by other forms of perception (e.g., Weber's law). Thus, even though our subjective experience of time might be quite different from the actual, physical duration of an event, temporal experience remains an important and necessary part of our everyday living and ultimately forms the fabric of our thoughts and behaviors.

Distortions in aspects of temporal processing from the range of milliseconds to seconds, to conceptual notions of a timeline for time (past, present, future) have been separately reported for a variety of psychological disorders. Aside from adages such as "a watched pot never boils" and "time flies when you're having fun," the experience of time distortions by the healthy individual may require oneself to imagine a world in which aspects of our temporal experiences are "off." For example, you have some sense of how long you've been reading this. Now imagine that this was suddenly uncertain for you (minutes, hours?). You would most likely feel anxious and aroused and would likely engage in different patterns of behavior and decision-making. Go a step further and imagine that you experience events that are disorganized in terms of order, duration, and sensory integration. How would that feel? There is no mental disorder of timing; and it is fair to say that deficits in timing are not characterizing deficits of any mental disorder. But it is also fair to say that the way in which aspects of temporal perception and sensitivity influence our psychology is profound, and there are many interesting findings implicating aspects of timing to the neurological, behavioral, and cognitive profiles characteristic of a number of mental disorders (many of which are described in this book!).

Perception, cognition, and action are tightly intertwined with events unfolding in time and, thus, many processes such as working memory, attention, decision making etc. are linked to timing, and implicated in successful interval timing models. Disturbance of one or more of these related processes could 
lead to time distortions or vice versa. Some researchers argue that certain disorders (e.g., dyslexia, aphasia) may potentially have their root solely on time distortions. For instance, Frederick T. Melges devoted his career to defining the role of time distortions in a number of psychiatric disorders. In his book, Time and the inner future, he supported that time disturbances distort a person's view of his/her future, thus disrupting goal-directed behavior and rendering the person unable to live a normal life.

It is widely believed that (within the bounds of the psychological present, 3-5-8 s) people with schizophrenia experience an "overload" of sensory input and lack the executive functioning skills to compensate and "sift-through" information. Schizophrenics often produce "word salads" and lose track of a train of thought, failing to link different sentences appropriately. Theories of autism also emphasize differences in "neural signatures," "temporal binding" or the adaptive functioning of long-range (multiple neural systems), and a form of sensory "overload" is posited with certain senses tending to become highly arousing (visual) or aversive (auditory, tactile). It has been shown recently that children with autism spectrum disorder reveal characteristic differences in supra-second timing, "bind" sensory information over an extended time window, and the relative functioning of aspects of temporal processing might correspond to characteristic features of the disorder (e.g., language, sensory processing).

The neural basis of timing and time perception is currently a "hot topic" in empirical psychology, but the extent to which the clinical features of certain disorders are related to time distortions per se is currently unknown, although this is beginning to be elucidated. Time perception in the secondsto-minutes range recruits cortico-striatal and cortico-cerebellar circuits (including various neurotransmitter systems) - brain circuits embedded in the frontal lobes and the basal ganglia, which are modulated by the dopamine transmitter system. Thus, patients with frontal lobe injuries display impaired estimation of time intervals, while patients with Parkinson's disease show decreased dopaminergic function in the basal ganglia accompanied by interval discrimination deficits. In the millisecond range, patients with dyslexia, lesions in the left hemisphere or aphasia, reveal various temporal processing difficulties; e.g., order distortions in aphasia, severe deficits in time perception and motor timing of short intervals in individuals with cerebellar damage, and a processing deficit for rapidly presented speech and non-speech stimuli in dyslexia. Children with attention deficit hyperactivity disorder and adults with aberrant impulsive behaviors, tend to overestimate and under-produce given time intervals, and appear less tolerant of delays in the imagined "future" - as if they consider them "too long." Time 
perception is also crucial in decision-making and impulse control. For example, a side effect of L-Dopa treatment for Parkinson's disease can be to increase impulsivity (and inappropriate behaviors) and the subjective 'warping' of time. This relationship of impulsivity and time perception has been attributed to an increased cognitive processing speed (and, thus, increased clock speed), given research on time perception showing that increased arousal often leads to interval overestimations. Disturbances in cognitive functioning and emotional processing can also modulate ones temporal percept.

In testament to the multiplicity of temporal processing to the human condition, we also think about the consequences of our actions in hypothetical time; discount events based on imagined delay; and temporal dynamics influence our valuations of future rewards and past experience. As it may relate to human's capacity for 'mental time travel', and the extent to which this forms the scaffold for our lives, there is some historical basis for positing there may be fundamental pathophysiological differences in certain clinical (psychiatric, psychological, neurological) populations. Disorientation in time and inability to coordinate and orientate oneself temporally in the external world is routinely observed in the majority of neurological patients, and those with states of mental confusion (e.g., intellectual disability, dementia), who also present with poorly developed abstract concepts of time.

The problem for modern psychiatry as a form of medicine is that it has a nomenclature of mental illness, but little known etiologies. The mind is considered an emergent property of the brain, not a "product" like urine from a kidney. The presence of mind is based on the interactions of elements in the brain, and the adequate organization of elements is fundamental for all conscious mental features (faculties, drives, responses). One of the first psychiatrists to characterize time in psychopathological minds was Eugene Minkowski. Minkowski proposed that individuals with schizophrenia lack a 'feeling of time': They understand 'before' and 'after', but live very much in the 'present'. Melges described paranoid psychiatric patients as having a form of "temporal disintegration" - memories of the past, perceptions in the present, and expectations in the future may be confused and appear to be happening at the same time, thus they may appear interconnected. Feelings of sadness, Minkowski noted, often accompany thoughts of the past, and people suffering from depression and compulsive neuroticism may tend to "live in the past," while neurotics in general tend to neglect the "present." People who are depressed tend to overestimate intervals, possibly due to increased allocation of attention to the passage of time (or rumination), which in turn leads to a higher processing of time units and, thus, overestimation of an event. It has been 
reported that children with autism also experience difficulty thinking forwards and backwards in time.

As of today, we might not be able to claim that certain disorders are due to time distortions per se, but experimentation, imaging, and modeling allow us to better understand how timing is represented in the normal and disordered brain and mind. Recently, there has been a burgeoning interest in timing and time perception in certain disorders with a known neuropathology in identified interval-timing regions (Parkinson's disease, aphasia) or by virtue of diagnostic psychopathological distortions in the apparent temporal organization of cognition or behavior (e.g., attention deficit hyperactivity disorder, autism, schizophrenia). Deviations from "normal" timing patterns have been obtained with a variety of targeted psychological, psychiatric, and neurological patient populations on a variety of timing and time perception tasks. Timing is critical in everyday functioning and a crucial factor for studying (and maybe rehabilitating) the disturbed brain and mind.

It is pertinent, therefore, to collect the current knowledge on temporal processing in certain clinical populations. This book serves as such a collection, bringing together new and old research and ideas on aspects of temporal processing in clinical populations, in the ultimate hope that it will elucidate the interdependence between perturbations in timing and disturbances in mind and brain. The book as a whole does not provide the answer to the relative function of temporal processing within these populations (although this is reviewed). Instead it offers a window into cutting edge research examining temporal processing in these disorders, and as it relates to how these disorders present (their phenotypes). This collection of accumulated knowledge will be an excellent reference for the student and scientist interested in the topic of temporal processing, and abnormal psychology, but it will also serve as the stepping-stone to share ideas and push forward the advancement in understanding how distorted timing can lead to a disturbed brain and mind or vice versa.

Time Distortions in Mind - Temporal processing in clinical populations was inspired from the TIMELY (COST Action TDog04) Training School on "Temporal Processing in Clinical Populations" that was held in Thessaloniki, Greece on March 26-29, 2012. We would, thus, like to thank the TIMELY network and COST funding for supporting this endeavor and our authors who contributed to this book and patiently waited for its completion and publication. We would also like to dearly thank two people: Özlem Altin, who provided us with the cover art for this book (we encountered this work in a gallery in Berlin and immediately wished for it to be the book's cover art; Özlem was gracious to immediately reply to our email request and work with us in terms of the 
image's quality and presentation), and Mary Kostaki, who worked on the design of the cover for this book (and showed patience to all the changes - big or small - so as to reach the appropriate result for this excellent collection of chapters).

Melissa J. Allman and Argiro Vatakis 


\section{List of Figures}

4.1 Schematic representation of experimental design. During the "easy" condition, the 20oms standard tone was followed by a comparison tone of $70,100,300$ or $330 \mathrm{~ms}$ ( \pm 50 or $65 \%)$. During the "difficult" condition, the comparison tone was $160,170,230$ or $240 m s$ ( \pm 15 or $20 \%$ ). During "baseline," subjects pressed a button, but did not judge, following two 20oms tones. Reprinted from Schizophrenia Research, Vol. 127, D.B. Davalos, D.C. Rojas and J.R. Tregellas, Temporal processing in schizophrenia: Effects of task-difficulty on behavioral discrimination and neuronal responses, pp. 123-130. 102

4.2 Temporal processing during "easy" temporal processing. Reduced activation in the schizophrenia group relative to controls was observed in the supplementary motor area (SMA), insula/opercular cortex, and the dorsolateral prefrontal cortex (DLPFC). Statistical parametric maps thresholded at $\mathrm{p}<0.01$, overlaid onto the average T1-weighted anatomy of all subjects. Reprinted from Schizophrenia Research,Vol. 127, D.B. Davalos, D.C. Rojas and J.R. Tregellas, Temporal processing in schizophrenia: Effects of taskdifficulty on behavioral discrimination and neuronal responses, pp. 123-130. 104

4.3 Temporal processing during "difficult" temporal processing. Reduced activation in the schizophrenia group relative to controls was observed in the SMA, insula/ opercular cortex, and the DLPFC, as well as the striatum and thalamus. Statistical parametric maps thresholded at $\mathrm{p}<0.01$, overlaid onto the average T1-weighted anatomy of all subjects. Reprinted from Schizophrenia Research,Vol. 127, D.B. Davalos, D.C. Rojas and J.R. Tregellas, Temporal processing in schizophrenia: Effects of task-difficulty on behavioral discrimination and neuronal responses, pp. 123-130. 104

4.4 "Difficult" compared to "easy" temporal processing. Reduced activation in the schizophrenia group relative to controls was observed in the insula/opercular cortex and the striatum. Statistical parametric maps thresholded at pbo.o1, overlaid onto the average T1-weighted anatomy of all subjects. Reprinted from Schizophrenia Research,Vol. 127, D.B. Davalos, D.C. Rojas and J.R. Tregellas, Temporal processing in schizophrenia: Effects of task-difficulty on behavioral discrimination and neuronal responses, pp. 123-130. 105

5.1 Illustration of the paradigm used to check for a non-specific effect of subjective judgments. The curves represent the increase in luminance of the two target bars, A and B. The first increase in luminance is used as a prime and masked by the distracters ('priming' figure). The prime is asynchronous when the two bars increase their luminance asynchronously. The participant's task is to decide 
whether the second increase in luminance is simultaneous or asynchronous. 120

5.2 Illustration of the procedure designed to compare simultaneity/asynchrony discrimination for connected and unconnected squares and squares displayed in the same hemifield vs. in different hemifields. Two squares are filled in, in gray, either simultaneously or asynchronously. These two squares are either connected or not. Participants are instructed to hit the right key when they think the squares are filled in asynchronously and the left key when they think the filling occurs simultaneously. In the examples, filled squares are connected and located within the same hemifield in the right top figure and unconnected and in different hemifields in the right lower figures. The intra- vs. across-hemifield manipulation has been conducted without connecters in a first experiment (Lalanne et al., 2012b) and with connecters in a second, distinct experiment (Lalanne et al., 2012c). The examples correspond to the second experiment. In this experiment, the location of the connecters (vertical vs. horizontal) and the location of the targets (within the same vs. different hemifields) yielded four main possibilities (targets connected within the same or different hemifields and targets unconnected within the same or different hemifields), which were equally represented and displayed in random order. 124

5.3 Illustration of the successive events in the simultaneity/asynchrony discrimination task in case of an asynchrony (from left to right). When 4 locations are used (upper row) and the first square is filled in (middle figure in the upper row), there are two possible locations for the second one (figure on the right), and there is, thus, an uncertainty regarding the location of the second stimulus. The spatial location of the second stimulus is always predictable when only two locations are used (lower row). These two experiments have been conducted in two different groups of participants (Lalanne et al., 2012c). 125

5.4 Amplitude of the bias (in \%) to the side of the 1st or 2nd stimulus. A negative bias corresponds to a bias to the side of the first stimulus (in patients), whereas a positive bias corresponds to a bias to the side of the second stimulus (in healthy participants). $\quad 126$

5.5 Illustration of the experimental conditions used in our location priming paradigm. Priming frames were either simultaneous or asynchronous with a sOA of $17 \mathrm{~ms}$ between frames. One of the frames was then filled in and participants had to press a response key to the side of the target. The results correspond to a soA of 10oms between prime frames and the target. 127

5.6 Illustration of the experimental conditions used in our succession priming paradigm. Priming frames were either simultaneous or asynchronous with a 
SOA of $17 \mathrm{~ms}$ between frames. The two frames were then filled in with an asynchrony of 10oms, with these filled in squares representing the targets. Participants had to press a response key to the side of the 2nd target. The results correspond to a SOA of 100ms between prime frames and the targets. 128

6.1 Picture of the experimental apparatus used to measure squeeze forces produced on the load cell with the participants' dominant hand (example of a left-handed patient with schizophrenia) in function of a metronome (left). Example of an equivalent rhythm for which subjects were required to alternate force levels in function of high and low pitch tones. Note the difficulty in force alternation but also of the presence of an attentional lapse twelve seconds after the start of the trial (right). 146

6.2 Number of bad trials measured in healthy controls (black) and patients with schizophrenia (grey) in a synchronisation task requiring subjects to perform a series of squeezes on a load cell following with equivalent time intervals (Ro; $\mathrm{R} 2)$ and alternated time intervals $\left(\mathrm{R} 1 ; \mathrm{R}_{3}\right)$ with equivalent force levels (Ro; $\left.\mathrm{R} 1\right)$ or alternated force levels $\left(\mathrm{R}_{2} ; \mathrm{R}_{3}\right)$. Results showed that for both controls and patients, it was difficult to alternate force levels and maintain the timing motor task but patients failed more often than the controls (top). When assessing absolute timing errors on those trials performed correctly (bottom), results demonstrated that patients made significantly more timing errors than controls in those conditions for which alternated time intervals were to be performed. Overall, these results suggest a specific difficulty in predictive timing in schizophrenia. 148

6.3 Graphical representation of the errors in body schema observed in patients with schizophrenia (red) and controls (blue) during the arm "return" paradigm at four critical moments: $0,3,5,8$ minutes from the start of the experimental session. Note that reproduction errors of hand symmetrical position compared to the subjects' body sagittal axis increases at a similar rate for both subject groups suggesting a normalised ticking clock decay in schizophrenia. $\quad 151$

6.4 Illustration of the experimental setup used to measure spontaneous tempo in controls and patients with schizophrenia (left). Results showed that tempo was slower in patients but this difference was solely due to a significant increase in contact duration (CD) of finger on the screen. Flight time (FT) was similar across groups arguing in favour of a normalised ticking frequency of the internal clock in schizophrenia (right). 153

6.5 Results obtained in the Spatio-tapping task in controls (grey) and patients with schizophrenia (black). The top figure (6.5a) illustrates the inter-tap interval error (ITI in ms) compared to the target inter response interval (IRI in ms) that was imposed by a regular metronome. The middle figure $(6.5 \mathrm{~b})$ presents that mean results obtained for the Contact times (Ст in ms) measured for the 11 
different tempi. Finally, the bottom figure $(6.5 \mathrm{c})$ presents the mean orientation of the endpoint error distributions calculated for oriented scatter plots towards the next target. Large values suggest an absence of orientation. $\quad 159$

7.1 The upper panels show the genetic system used to generate the $\mathrm{D}_{2} \mathrm{R}-\mathrm{OE}$ mice. In one mouse line, the human $\mathrm{D} 2$ receptor gene is expressed under control of the tetO promoter. In a second mouse line, the tTA element is expressed under control of the CamKIIa promoter. In a mouse in which both transgenes are present, the tTA element binds to the tetO promoter, driving expression of D2 receptors. In the $\mathrm{D}_{2} \mathrm{R}-\mathrm{OE}$ mice, expression of excess $\mathrm{D}_{2}$ receptors is confined to the striatum and olfactory tubercule (shown via in situ hybridization in the upper right panel). Feeding the mouse doxycycline prevents tTA from binding to the tetO promoter (bottom left panel), thereby eliminating expression of the transgenic D2 receptors (bottom right panel). $\quad 171$

7.2 Temporal information processing during the peak procedure. Responses per second as a function of time during peak interval trials (see text for details). D2R-OE mice have lower response rates, and are less accurate and precise in their timing of the $24 \mathrm{~s}$ target interval. Turning off the transgene (D2R-OE-Dox) increases response rates and rescues accuracy of interval timing. Data taken from Drew et al. (2007). 173

7.3 Assessment of motivation in the progressive ratio in $\mathrm{D}_{2} \mathrm{R}-\mathrm{OE}$ mice. A. Survival functions showing the percentage of mice in all groups that were still responding on the schedule as a function of time in the progressive ratio session. $\mathrm{D}_{2} \mathrm{R}-\mathrm{OE}$ mice quite responding significantly sooner than controls, and turning off the transgene (D2OE-Dox) rescues the progressive ratio performance. B. Number of rewards earned and break point (last ratio completed) for all groups of mice. D2R-OE mice earned significantly fewer rewards and had significantly lower break points that control and D2R-OE-Dox mice. C. Total number of lever presses emitted by all groups of mice on the progressive ratio schedule. $\mathrm{D}_{2} \mathrm{R}-\mathrm{OE}$ mice made significantly fewer lever presses that control mice. Turning off the transgene improved performance. D. Number of head entries made by all groups of mice following a reward delivery. There was no difference in this measure across groups, indicating that all mice consumed the reward once it was earned. Reprinted with permission from Drew et al. (2007). 174

7.4 A. Peak interval performance of both control and $\mathrm{D}_{2} \mathrm{R}-\mathrm{OE}$ mice is sensitive to manipulation of motivation. Motivation was manipulated by changing the percentage of rewarded fixed interval trials (see text for details). Circles show performance under conditions of $100 \%$ rewarded Fi trials, while triangles show performance under $10 \%$ rewarded Fi trials. Closed symbols show control performance, while open symbols show performance of D2R-OE mice. Decreasing motivation in control mice results in performance 
that is indistinguishable from that of $\mathrm{D}_{2} \mathrm{OE}$ mice. B. Performance of control and $\mathrm{D}_{2} \mathrm{OE}$ mice on the bisection procedure (see text for details) with anchor durations of 2 and $8 \mathrm{~s}$. The figure shows the proportion of trials on which the mice chose the response lever corresponding to a "long" sample duration as a function of sample duration. There are no differences in performance between genotypes. C. Performance of control and D2R-OE mice on the bisection procedure with anchor durations of 6 and $24 \mathrm{~s}$. $\mathrm{D}_{2} \mathrm{R}-\mathrm{OE}$ mice are selectively impaired on longer duration sample trials, suggesting a deficit in working memory or sustained attention. Data from Ward et al. (2009). 175

7.5 Conceptual framework showing the psychological processes which are thought to underlie temporal information processing, and their interaction with motivation (see text for details). 179

8.1 Interval timing models and brain networks. A: Illustration of the three component processes of an information processing model of timing, scalar expectancy theory (SET). The clock component consists of a pacemaker that represents time through the accumulation of pulses. Pulses are turned on and off by a switch and then passed into an accumulator to be counted. Accumulated pulses are encoded into working memory and over time, more enduring interval representations are stored in long-term memory. Decision processes compare pulse counts from the accumulator with ones in memory to determine when or how to respond. B: The neuroanatomically connectivity of the striatum and cortex is illustrated in the context of the Striatal Beat Frequency Model (SBF). By this model, the striatum receives cortical oscillatory activity, which evolves as a function of event duration, thereby serving as a clock signal. The striatum detects the state of the cortical oscillatory patterns and responds when a pattern matches previously reinforced or intrinsically important states, thereby serving as a core timer. Tonic dopamine and glutamate alter cortical oscillation frequencies; nigrostriatal phasic dopamine signals the onset and the offset of a timed event. Dopamine also strengthens striatal synapses that are activated by the frequency pattern of cortical neurons to a timed event. C: Illustration of anatomical pathways that enable two-way communication between the striatum and cerebellum. ACC $=$ anterior cingulate cortex; DLPFC = dorsolateral prefrontal cortex; $\mathrm{DN}=$ dentate nucleus; $\mathrm{FEF}=$ frontal eye fields; $\mathrm{GPe}=$ globus pallidus externa; $\mathrm{GPi}=$ globus pallidus interna; $\mathrm{PPC}=$ posterior parietal cortex; $\mathrm{PM}=$ premotor; $\mathrm{PN}=$ pontine nucleus; SMA = supplementary motor area; $\mathrm{SC}=$ sensory cortex; $\mathrm{SNC}=$ substantia nigra pars compacta; $\mathrm{SNr}=$ substantia nigra pars reticulata; $\mathrm{STN}=$ subthalamic nucleus; VLPFC = ventrolateral prefrontal cortex; VTA = ventral tegmental area. $\quad 193$ 
8.2 Time perception paradigm. A: Illustration of the trial events in the time perception task. A standard and a comparison interval were successively presented and separated by a delay. The standard was $1200 \mathrm{~ms}$ or $1800 \mathrm{~ms}$ and respectively pegged to a delay of $6800 \mathrm{~ms}$ or $6200 \mathrm{~ms}$. Three shorter and three longer comparison intervals (CI) were $\pm 7 \%$ increments of each si. Intervals were designated by filled tones or a blue sphere. B: The three hypothetical time-course functions illustrate the expected MR signal associated with encoding the standard interval (black curve), encoding the comparison interval (solid gray curve), and making a response (dotted gray curve). Arrows leading from each trial event designate their onset. The hemodynamic response peaks 4 to $6 \mathrm{~s}$ after the onset of the events. An image of the entire brain is acquired every $2 \mathrm{~s}$. The fixation cross is displayed throughout the task. Figure adapted from Harrington and colleagues. 204

8.3 Regional analyses of brain activation in PD for the encoding and decision phases. Functional regions of interest (ROI) were derived by conjoining timing-related activation from the control and PD groups. Blue areas blue signify regions in which activation did not differ between the groups. Red areas showed hypoactivity in the PD OFF group relative to the control group. Brain activation is projected onto the lateral (row 1) and medial (row 2) surfaces of the left and right hemispheres, the anterior and posterior surfaces of the cerebellum (row 3), and the left and right basal ganglia (row 4). Brain sections are displayed in neurological view. 206

8.4 Cortical regions showing dopamine-modulated connectivity with the striatum during the decision phase. For each striatal seed region, effective connectivity of the putamen and caudate is illustrated on axial or sagittal brain sections, which are displayed in neurological view. Striatal connectivity was stronger OFF than ON medication with elements of the motor circuit (green areas), the frontoparietal working-memory network (purple areas), and the limbic system (insula). Striatal connectivity was stronger ON than OFF medication with the superior frontal gyrus. IP = inferior parietal cortex; M FG = middle frontal gyrus; SFG = superior frontal gyrus; SMA = supplementary motor area. Figure adapted from Harrington and colleagues. 209

8.5 Regions showing abnormal activation during motor timing in prodromal hd. Top: Map of the functional regions of interest, which were derived from a conjunction map that was used to test for group differences in signal intensity. Green line in the sagittal image is perpendicular to the anterior-posterior commissure. $\mathrm{Z}$ coordinate $=\mathrm{mm}$ superior to the anterior commissure -posterior commissure line. Bottom: The graphs display the percent MRI signal change for the control, far, and CLOSE groups. Three different patterns of activation were uncovered: (1) far > Controls = CLOSE; (2) Control $>$ far $>$ CLOSE; and (3) 
far $=$ Controls $>$ CLOSE. Figure adapted from Zimbelman and colleagues (2007). $\mathrm{B}=$ bilateral hemispheres; $\mathrm{L}=$ left hemisphere; $\mathrm{R}$ = right hemisphere. $\quad 211$

8.6 Regions showing abnormal activation volume during time discrimination in prodromal HD. Top: Activation foci $(\mathrm{p}<.01)$ were derived from the timing minus control task comparison for the control, far, and CLOSE groups. $\mathrm{L}=\mathrm{left}$ hemisphere; $\mathrm{R}=$ right hemisphere; $\mathrm{z}$ coordinate $=\mathrm{mm}$ superior to the anterior commissure -posterior commissure line. Bottom: Volume of activation in the thalamus, caudate/putamen, and the preSMA/cingulate. Figure adapted from Paulsen and colleagues (2004). 214

9.1 Coefficient of variation (standard deviation divided by mean movement time) for repetitive finger tapping, discrete circle drawing and continuous circle drawing. Movements were performed by individuals with unilateral cerebellar lesions with both their impaired and unimpaired limbs. Error bars represent standard error. 234

9.2 (A) The Reset Task described by Karmarkar and Buonomano (2007). Participants judged the length of the target (T) relative to a previously presented standard interval when the target was presented without a distractor (top), with a fixed interval distractor (D; middle), or a variable length distractor (bottom). (B) Consistent with Karmarkar and Buonomano (2007), we found an increased threshold with a variable distractor condition for the 100-100 condition. This was not the case in the 100-300, 300-300, or 300-100 conditions (adapted from Spencer, Karmarkar, and Ivry, 2009). 240

10.1 Scalar Expectancy Theory (Gibbon, 1997; Gibbon et al., 1984). 251

11.1 Relative frequency distributions for the two target durations (8s and 21s) showing accuracy and precision of duration reproduction in Parkinson's disease (PD) patients ON and OFF their levodopa medication. $\quad 288$

11.2 Relative frequency distributions for a 21-s target duration plotted as a function of whether it was trained in conjunction with a 8-s target duration or by itself (21 only). The peak functions illustrate the accuracy and precision of duration reproduction in Parkinson's disease (PD) patients ON and OFF their dopaminergic medication as a function of the multiple or single target duration training. $\quad 289$

11.3 Proportion maximum response rate for 8-s and 21-s target durations for Parkinson's disease (PD) patients trained ON and OFF their levodopa medication. Data are plotted in time relative to the median time and normalized as proportions of the maximum response rate/frequency. Peak functions superimpose in the ON medication state, but not in the OFF medication state - indicating a violation of the scalar property of interval timing with lowered dopaminergic function. 291 
11.4 Peak times (means $\pm \mathrm{SE}$ ) for participants in the control (CON) and haloperidol (HAL) conditions tested with 7-s and 14-s target durations in the peak-interval procedure. Data are plotted as a function of the probability of intertrial interval (ITI) feedback $(25,50$, and $100 \%) . \quad 296$

11.5 Peak times (means $\pm \mathrm{SE}$ ) for Parkinson's disease (PD) patients trained ON and OFF their dopaminergic medication and aged-matched controls using 7 -s and 14-s target durations in the peak-interval procedure. Data are plotted as a function of the probability of intertrial interval (ITI ) feedback $(25,50$, and $100 \%) .298$

11.6 Ordinal comparison task using hue and duration stimulus combinations in the manner of Coull et al. (2004). The duration data presented here represent the grand average response-locked event-related potentials (ERPS) at electrode Fz after correct left-handed (green) and right-handed (red) responses and incorrect left-handed (magenta) and right-handed (cyan) responses in patients with Parkinson's disease (PD) in the ON and OFF medication states. The o-ms time point represents the time of the "shorter" or "longer" classification (key press) determined by a left-handed or right-handed response. The vertical line represents the peak latency of the error related negativity (ERN) in the PD patients. The ERN is smaller in the OFF medication state than in the ON medication state for the left-handed (magenta) incorrect response with no difference in the Pe between conditions (see Falkenstein et al., 2001, 2005). Interestingly, no handedness/lateralization effects were observed for the hue condition in the PD patients as a function of the ON/OFF states. 303

11.7 Bayesian simulations of 7-s and 14-s target durations in the peak-interval procedure for Parkinson's disease (PD) patients tested ON and OFF dopaminergic medication. Weber fractions for sensory measurement $(\mathrm{Wm})$ were set as 0.1 in PD-ON medication state and 0.3 in PD-OFF medication state. Simulation of 200,000 trials demonstates veridical timing (i.e., response functions centered on the target durations) in the PD-ON medication state and a high degree of migration (i.e., overestimation of the shorter duration and underestimation of the longer duration - Vierordt's law) for the 7- and 14-s target durations in the PD-OFF medication state (left panel). Scaled distribution functions, i.e., proportion of maximal responding plotted on a relative time scale (right panel), show that the estimations of 7 - and 14-s target durations superimpose on top of each other in the PD-ON medication state. In contrast, the response function for the 14-s target duration shows a narrower dispersion compared to the response function for the 7 -s target duration in the PD-OFF medication state, indicating a violation of scalar property of interval timing (see Hinton \& Rao, 2004; Malapani et al., 1998; Rakitin et al., 1998). 308 
12.2 Auditory order thresholds (mean and standard deviations) for the five patient groups with focal brain injuries and for an orthopaedic control group are shown: LH. pre-anterior left hemisphere (pre-central) with non-fluent aphasia; L H. post-posterior left hemisphere (post-central) with fluent aphasia $\left({ }^{*} \mathrm{p}<1 \%\right.$ for group differences as compared with controls and L. noAph; statistical calculation with Scheffe post-hoc test); L. noAph-left-sided subcortical lesions without aphasia; rh. pre-anterior right hemisphere (pre-central); R H. postposterior right hemisphere (post-central). Reprinted from Neuroscience Letters, 264, v. Steinbüchel N., Wittmann M., Strasburger H., and Szelag E. "Auditory temporal-order judgment is impaired in patients with posterior regions of the left hemisphere" $168-71$, copyright (1999) with permission from Elsevier. $\quad 349$

12.3 The measured integration interval length (MIIL) plotted against the metronome frequency using three different strategies in Broca's aphasia and other patients. Integration (A) by time; (B) by number; (C) in Broca's aphasics; (D) in all the remaining patients. Standard deviation values (in $\mathrm{ms}$ ) for the consecutive frequencies in the Broca's aphasics: 1964, 911, 887, 591, 439, 363, 408, 491, 406; in the other subjects: $1191,698,535,370,338,370,363,394,401$. Reprinted from Neuroscience Letters, 235, Szelag E. v. Steinbüchel N., Pöppel E. “Temporal processing disorders in patients with Broca's aphasia” 33-36, copyright (1997) with permission from Elsevier. 350

12.4 Maximum-tapping tempo and personal-tapping tempo as mean inter-response interval (IRI) over the orthopaedic control group and the brain-injured patient groups with left-hemispheric cortical lesions $(\mathrm{LH})$, with left-hemispheric lesions in predominantly subcortical regions (LH sub) and with right-hemispheric cortical lesions ( $\mathrm{RH}$ ). Data for the brain-injured patients are only presented for the hand ipsilateral to the lesion site. Reprinted from Cognitive Brain Research, 10, Wittmann M., v. Steinbüchel N., Szelag E. "Hemispheric specialisation for self-paced motor sequences" 341-44, copyright (2001) with permission from Elsevier. $35^{2}$

13.1 A schematic and simplified representation of the role of the suprachiasmatic muclei $(\mathrm{SCN})$ of the mammalian brain as the primary circadian pacemaker. The action of light on melanopsin containing cells of the retina can reset the phase of the 24 h oscillations in neural activity within the SCN. The SCN regulates (black solid arrows) the production of certain hormones such as melatonin and cortisol that show characteristic variation in level over the day-night cycle. Cortisol and melatonin convey information of circadian phase to peripheral clocks and, thus, couple the timing of metabolic processes and behaviours to sCN time. 359 
13.2 A schematic representation of the interaction between Neuronal Homeostatic Limit (grey box) of expression of neuronal genes and circadian regulation of one of these neuronal genes (sinusoid); graphs A, B, and C. A, neurotypical; $B$, variation causing a circadian signal with increased amplitude; $C$, variation causing increased base line (constitutive) expression of clock controlled gene. D represents another clock-controlled gene where neurotypical circadian boosting (solid line) fails due to mutation that produces a week circadian signal (dashes). $\quad 372$ 


\section{List of Tables}

1.1 A brief overview of all the studies reviewed in this chapter covering issues related to multisensory temporal processing $\quad 22$

2.1 Details and results of the studies on timing studies in asd and healthy controls 39

4.1 MNI coordinates and statistics for brain regions with greater activation in controls compared to individuals with schizophrenia. Reprinted from Schizophrenia Research, Vol. 127, D.B. Davalos, D.C. Rojas and J.R. Tregellas, Temporal processing in schizophrenia: Effects of task-difficulty on behavioral discrimination and neuronal responses, pp. 123-130. Copyright $2011 \quad 103$

6.1 Mean values (standard deviation) obtained in healthy controls and patients with schizophrenia for the tempo of spontaneous tempo, with the subdivision of inter-response-intervals (IRI) in Contact Durations (CD in ms) and Flight Times (FT in ms). Note that patients are as regular than controls in their rhythmic production 154

10.1 Studies of perceptual timing in patients with Parkinson's disease (PD) compared to healthy controls, organized by type of task 257

10.2 Studies of the synchronization-continuation task in patients with Parkinson's disease (PD) compared to healthy controls 263

10.3 Studies of perceptual timing in patients with lesions of the frontal lobe compared to healthy controls, organized by type of task 269

11.1 Temporal migration effects in Parkinson's Disease 293

11.2 Bayesian model predictions for peak-interval measurements 309

12.1 The summary of main language deficits observed in particular aphasic syndromes 331

12.2 Summary of results reported in the existing literature regarding deficient temporal processing in aphasic patients 336

13.1 Conserved circadian control elements are not present in genes that affect interval timing $\quad 367$ 